\title{
Evaluation of Perioperative Complications in the Management of Biliary Atresia
}

\author{
Min $\mathrm{Du}^{\dagger}$, Junfeng Wang ${ }^{\dagger}$, Yue Tang, Jingying Jiang, Gong Chen, Yanlei Huang, Zhen Shen, \\ Rui Dong* and Shan Zheng*
}

Department of Pediatric Surgery, Children's Hospital of Fudan University, and Shanghai Key Laboratory of Birth Defect, Shanghai, China

\section{OPEN ACCESS}

Edited by:

Francesco Morini,

Bambino Gesù Children Hospital

(IRCCS), Italy

Reviewed by:

Daniele Alberti,

University of Brescia, Italy

Tetsuya Ishimaru,

Saitama Children's Medical

Center, Japan

*Correspondence:

Rui Dong

rdong@fudan.edu.cn

Shan Zheng

szheng@shmu.edu.cn

†These authors have contributed equally to this work and share first authorship

Specialty section:

This article was submitted to

Pediatric Surgery,

a section of the journal

Frontiers in Pediatrics

Received: 22 April 2020

Accepted: 30 June 2020

Published: 28 August 2020

Citation:

Du M, Wang J, Tang Y, Jiang J, Chen G,

Huang $Y$, Shen Z, Dong $R$ and Zheng $S$

(2020) Evaluation of Perioperative Complications in the Management of

Biliary Atresia. Front. Pediatr. 8:460.

doi: 10.3389/fped.2020.00460
Purpose: To analyze the influence of perioperative complications in the management of biliary atresia (BA).

Methods: A retrospective study was performed using a total of 422 BA patients who underwent Kasai portoenterostomy (KPE) in a single institution between February 2016 and May 2017. Data on patients' clinical characteristics, laboratory examinations, perioperative complications, and outcomes were collected. Unpaired two-tailed $t$-test and $\chi^{2}$ test were employed for the comparison between BA patients with and without perioperative complications. Cox regression analysis was used to screen the risk factors for 2-years NLS in BA, and their influence on the 2-years NLS was analyzed using Kaplan-Meier survival analysis as well as the log-rank test.

Results: The incidence of perioperative complications, 6-months jaundice clearance (JC) and 2-years native liver survival (NLS) rate were 60.4, 59.5, and 56.6\%, respectively. Patients with perioperative complications had lower serum albumin (ALB) level, but higher aspartate aminotransferase-to-platelet ratio index (APRI) and international normalized ratio (INR) levels when compared with those without perioperative complications (ALB, $P<0.05$; APRI, $P<0.01$; INR, $P<0.05$ ). Moreover, perioperative complications were correlated with glucocorticoid administration $(P=0.002)$. Univariate Cox regression analysis showed no relationship between perioperative complications and 2-years NLS $(P>0.05)$. However, multivariate Cox regression analysis indicated 6-months JC was an independent protective factor for 2-years NLS $[P<0.0001$, hazard ratio $(H R)=0.074$, $95 \%$ confidence interval $=0.05-0.11]$, and concordance index of this prediction model including age, weight, APRI, glucocorticoid, and 6-months JC was 0.811.

Conclusion: Although perioperative complication is common during and after KPE, it had no influence on the prognosis of BA. However, assessment of the serum level of total bilirubin after KPE may serve as an important predictor for the outcome in BA.

Keywords: biliary atresia, glucocorticoid, jaundice clearance, native liver survival, perioperative complication 


\section{INTRODUCTION}

Biliary atresia (BA) is a life-threatening pediatric hepatobiliary disease that is caused by a progressive inflammatory and fibrotic obliteration of the bile ducts (1). The pathophysiology of BA is not yet clearly understood, and theories on the pathogenesis of BA include immunological factors, genetic predisposition, environmental toxins, and infection (2). In Asia, the BA incidence $(1 / 5,000-8,000$ live births) is considerably higher compared with that in Europe (1/15,000-20,000 live births) $(3,4)$. According to the anatomical structure, the Japanese Society of Pediatric Surgery classified BA into three types, including type I BA (choledochal atresia), type II BA (the common hepatic duct atresia), and type III BA (hepatic portal atresia) (5). Type III BA was previously considered as the "uncorrectable" type with poor outcomes affecting $80-95 \%$ of patients $(6,7)$.

When left untreated, BA patients will die of cirrhosis and hepatic failure within 2 years (8). Kasai portoenterostomy (KPE) is considered the first-line treatment for BA and restores bile flow from the liver to the intestine (9). In previous studies, it was demonstrated that native liver survival (NLS) rates ranged from 20 to $76 \%$ at $1-3$ years following KPE (10). If KPE fails, liver transplantation is the definitive therapeutic option (11). Even in cases of successful KPE, complications occur in $\sim 60 \%$ of patients; however, large cohort studies that focus on the comprehensive perioperative complications of BA are limited $(12,13)$. Perioperative complications have previously been defined as complications that occurred during and up to 30 days after surgery (14). Pressing questions for surgeons to answer are as follows: What are the characteristics of perioperative complications of $\mathrm{BA}$ ? And what is the impact of perioperative complications on the outcome of BA? This study aimed to characterize perioperative complications of BA and analyze the predictors of prognosis, and the relationship between perioperative complications and prognosis in BA.

\section{PATIENTS AND METHODS}

\section{Establishment of the Retrospective Cohort}

This study was a retrospective study that was performed in Children's Hospital of Fudan University (Shanghai, China). The study was conducted in compliance with the Declaration of Helsinki and was approved by the institutional ethical review board. As type III BA was the most common and prone to poor outcomes, this study focused on type III BA. A total of 422 type III BA patients were enrolled. The follow-up endpoints were as follows: (1) 24 months after KPE; (2) patients passed away or underwent liver transplantation. Inclusion criteria were as follows: (1) surgical exploration and cholangiography that confirmed type III BA; (2) KPE was performed by the same operation team. Exclusion criteria were as follows: (1) patients who suffered from immunodeficiency, hemolytic jaundice, and life-threatening serious diseases involving other systems such as heart failure, respiratory failure, and kidney failure, and so on; (2) follow-up was <24 months; (3) data on perioperative complications and 2-years outcome were unavailable.

\section{Operative Technique and Postoperative Care}

The KPE procedure adopted the classic Kasai procedure with spur valve for antireflux, and the length of the jejunal Roux-enY loop was $30-35 \mathrm{~cm}$. Unified postoperative adjuvant therapies included antibiotics to prevent cholangitis (first 2 weeks after KPE: intravenous cefoperazone sulbactam, $100 \mathrm{mg} / \mathrm{kg} / \mathrm{d}$, every $8 \mathrm{~h}$, and ornidazole $20 \mathrm{mg} / \mathrm{kg} / \mathrm{d}$, every $12 \mathrm{~h}$; from 3 weeks to 6 months after KPE: sulfamethoxazole/trimethoprim, 25 $\mathrm{mg} / \mathrm{kg} / \mathrm{d}$, every $12 \mathrm{~h}$, and cefaclor, $20 \mathrm{mg} / \mathrm{kg} / \mathrm{d}$, every $8 \mathrm{~h}$, are taken alternately every 2 weeks), ursodeoxycholic acid (20 $\mathrm{mg} / \mathrm{kg} / \mathrm{d}$, twice a day), drugs of liver protection (glycyrrhizin, $25 \mathrm{mg} / \mathrm{d}$, twice a day), fat-soluble vitamins (vitamin $\mathrm{A}$ and D drops, 2,000 IU/d, every day; vitamin E $10 \mathrm{mg} / \mathrm{d}$, every day; vitamin $\mathrm{K}_{1}, 5 \mathrm{mg} /$ time, biweekly), and nutritional support. When insufficient bile flow was presented with gray-pigmented stool without any surgery-related complications, glucocorticoid (methylprednisolone starting at $4 \mathrm{mg} / \mathrm{kg} / \mathrm{d}$ and tapering down for 3 months) was used. Jaundice clearance (JC) was defined as total bilirubin (TBIL) $<20 \mu \mathrm{mol} / \mathrm{L}(15,16)$.

TABLE 1 | Baseline characteristics of the biliary atresia study cohort $(n=422)$.

\begin{tabular}{lc}
\hline Variable & Value \\
\hline Age $(\mathrm{d})(\bar{x} \pm \mathrm{s})$ & $62.9 \pm 18.4$ \\
Female, $n(\%)$ & $218(51.7)$ \\
Weight (kg) $(\bar{x} \pm \mathrm{s})$ & $4.7 \pm 0.8$ \\
Laboratory examinations $(\bar{x} \pm \mathrm{s})$ & \\
ALT (U/L) & $102.3 \pm 77.3$ \\
AST (U/L) & $169.4 \pm 123.5$ \\
ALB (g/L) & $38.5 \pm 5.3$ \\
TBIL ( $\mu$ mol/L) & $160.5 \pm 65.9$ \\
DBIL ( $\mu$ mol/L) & $109.6 \pm 96.7$ \\
GGT (U/L) & $682.8 \pm 554.3$ \\
APRI & $1.728 \pm 1.611$ \\
Hb (g/L) & $98.9 \pm 16.3$ \\
INR & $1.0 \pm 0.2$ \\
TBA ( $\mu$ mol/L) & $145.3 \pm 87.5$ \\
Extrahepatic malformation, $n$ (\%) & $47(11.1)$ \\
CMV infection, $n(\%)$ & $139(32.9)$ \\
Glucocorticoid, $n$ (\%) & $271(64.2)$ \\
1-month JC, $n$ (\%) & $30(7.1)$ \\
3-months JC, $n$ (\%) & $193(45.7)$ \\
6-months JC, $n$ (\%) & $251(59.5)$ \\
1-year JC, $n(\%)$ & $250(59.2)$ \\
2-years JC, $n(\%)$ & $221(52.4)$ \\
1-year NLS, $n(\%)$ & $329(78.0)$ \\
2-years NLS, $n(\%)$ & $239(56.6)$ \\
2-years LT, $n(\%)$ & $79(18.7)$ \\
2-years death, $n(\%)$ & $104(24.6)$ \\
\hline
\end{tabular}

$A L B$, albumin; $A L T$, alanine aminotransferase; $A P R I$, aspartate aminotransferase-toplatelet ratio index; AST, aspartate aminotransferase; CMV, cytomegalovirus; DBIL, direct bilirubin; GGT, $\gamma$-glutamyl transpeptidase; $H b$, hemoglobin; INR, international normalized ratio; TBA, total bile acid; TBIL, total bilirubin. 


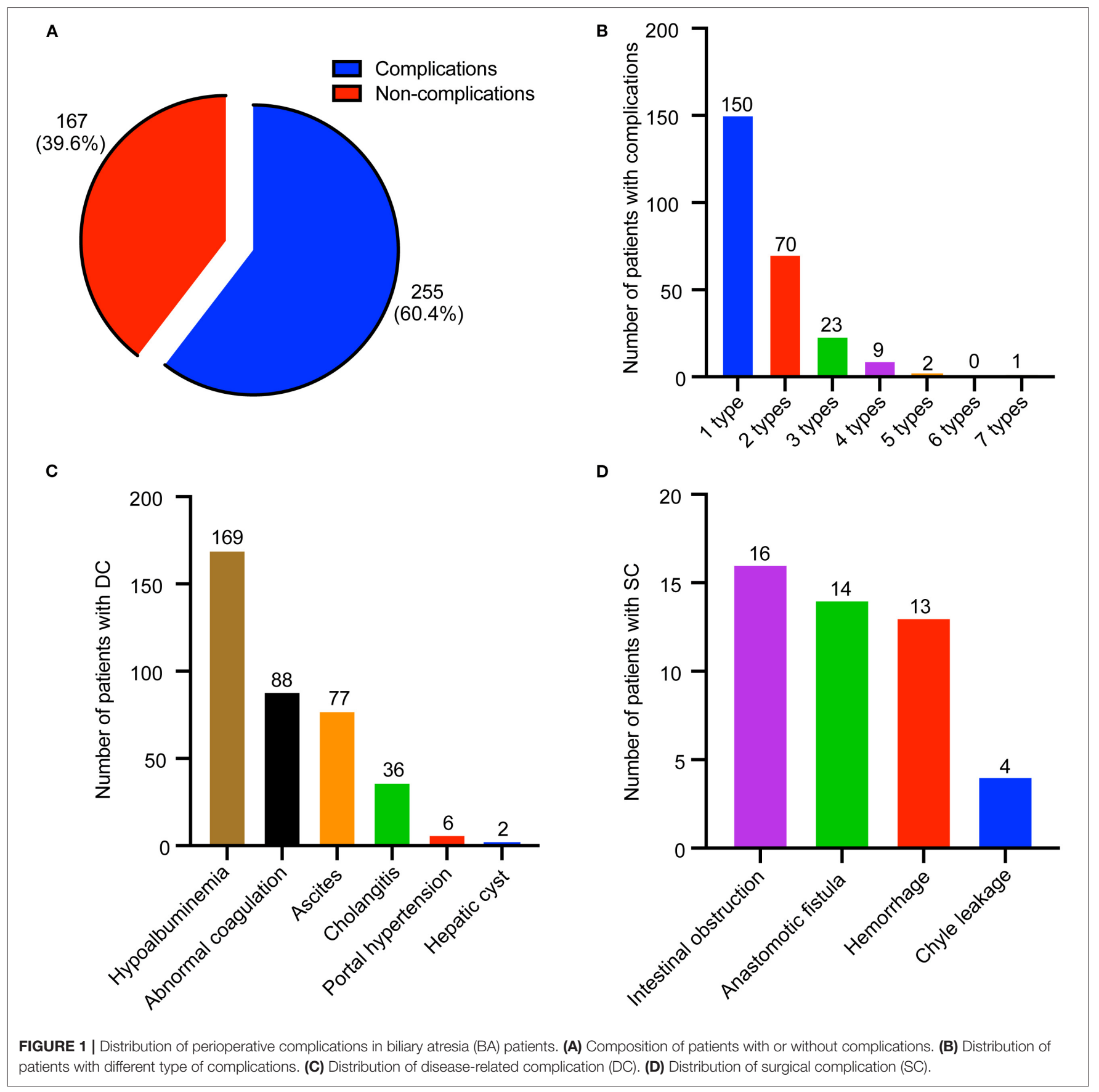

\section{Data Collection}

Baseline data were collected from each patient at the time of KPE, including gender, age, weight, the presence of cytomegalovirus $(\mathrm{CMV})$ infection [immunoglobulin $\mathrm{M}\left(\operatorname{IgM}^{+}\right)$], and extrahepatic malformations. The use of glucocorticoids and perioperative complications were acquired during and up to 30 days postKPE. Perioperative complications were classified into surgical complications (SCs) and disease-related complications (DCs). Surgical complications were defined as complications that were directly related to surgery, whereas DCs were defined as complications that were related to the underlying disease (17, 18). Disease-related complications included symptoms that were absent pre-KPE, but occurred after KPE, and can be classified as liver dysfunction (hypoalbuminemia, coagulopathy), ascites, cholangitis, portal hypertension (esophageal variceal bleeding), and intrahepatic biliary lakes (hepatic cyst) (18). Serum albumin (ALB) <30 g/L was considered hypoproteinemia (19). Abnormal coagulation was judged by the presence of prolonged prothrombin time (PT) or activated partial thromboplastin time (APTT), or higher international normalized ratio (INR) 
or decreased fibrinogen (FIB) (reference ranges: PT, 11-14.5 s; APTT, 26-40 s; INR, 0.8-1.2; FIB, 2-4 g/L). Cholangitis was diagnosed upon clinical symptoms (unexplained fever, recurrent jaundice, and acholic stools) and laboratory examinations (elevated white blood cell counts and C-reactive protein, and higher bilirubin levels) (20). Ascites and hepatic cyst were found by the abdominal ultrasonography post-KPE. From each patient, laboratory examinations were collected at KPE including TBIL, direct bilirubin (DBIL), ALB, aspartate aminotransferase, alanine aminotransferase, $\gamma$-glutamyl transpeptidase (GGT), total bile acid, aspartate aminotransferase-to-platelet ratio index (APRI), hemoglobin, and INR. The above parameters were reevaluated by collecting medical records during hospitalization and outpatient clinical records or telephone communication after discharge up to 30 days after KPE. The follow-up data included JC and NLS at 1 month, 3 months, 6 months, 1 year, and 2 years after KPE. Among them, 2-years NLS was set as the main prognostic indicator in this study.

\section{Statistical Analysis}

Statistical analyses were performed using SPSS software version 23.0 (IBM Corp., Armonk, NY, USA) and R software 3.6.0 (Lucent Technologies, Murray Hill, NJ, USA). Continuous data are presented as the mean \pm standard deviation, and categorical variables are presented as absolute numbers and percentages. Unpaired two-tailed $t$-test and $\chi^{2}$ test were employed for the comparison between BA patients with and without perioperative complications. Univariate Cox regression analysis was employed to choose the potential predictive factors of 2-years NLS of BA $(P<0.2)$, and then the prediction model was established by the above factors and was analyzed using multivariate Cox regression analysis. Results were expressed as the hazard ratio (HR) and 95\% confidence interval (CI). In addition, the NLS rate was analyzed using Kaplan-Meier survival analysis, as well as the log-rank test. Values were considered statistically significant when $P<0.05$.

\section{RESULTS}

\section{Baseline Data of the BA Study Cohort}

From February 2016 to May 2017, 422 type III BA patients were enrolled. The baseline characteristics at the time of KPE are presented in Table 1. The mean age was around 2 months, and the numbers of male and female patients were comparable. As expected, BA patients manifested hepatocellular damage and signs of obstructive jaundice with elevated DBIL, bile acids, and GGT. Approximately one-third of children were infected with CMV. A total of 51 extrahepatic malformations were detected in 47 patients (11.1\%), including cardiac defects (38 patients, 9.0\%), intestinal malformation (10 patients, $2.4 \%$ ), splenic malformation (two patients, $0.5 \%$ ), and visceral inversion (one patient, $0.2 \%$ ). To improve the insufficient bile flow, 271 patients (64.2\%) were administered glucocorticoids after KPE. The time to initial glucocorticoid treatment ranged from 5 to 60 days (median $=27$ days) after KPE. Among the population with glucocorticoids, a good bile drainage occurred in 95 patients (30.06\%), 135 patients (49.81\%), 136 patients $(50.18 \%)$, and
116 patients $(42.80 \%)$ at $3,6,12$, and 24 months after $\mathrm{KPE}$, respectively. In total, the rate of JC climbed to the highest (59.5\%) at 6 months after KPE and then fell slightly (52.4\%) at 2 years after KPE. A total of 239 patients (56.6\%) of BA patients survived with native liver; the rest comprised those with liver transplantation (18.7\%) and death $(24.6 \%)$ at 2 years after KPE. During the perioperative period, a total of three patients $(0.7 \%)$ passed away. One patient who underwent KPE at the age of 136 days died of liver failure. Another patient who underwent KPE at the age of 45 days died of gastrointestinal bleeding due to portal hypertension. A third patient at day 38 after KPE suffered from intestinal obstruction and abnormal coagulation and died after her parents insisted on giving up treatment.

TABLE 2 | Matched factors of biliary atresia patients with perioperative complications or not.

\begin{tabular}{|c|c|c|c|}
\hline \multirow[t]{2}{*}{ Variable } & \multicolumn{2}{|c|}{ Perioperative complications } & \multirow[t]{2}{*}{$P$ value } \\
\hline & No $(n=167)$ & Yes $(n=255)$ & \\
\hline Age, $d(\bar{x} \pm s)$ & $62.5 \pm 17.6$ & $63.2 \pm 19.0$ & 0.700 \\
\hline Female, $n(\%)$ & $82(49.1)$ & $136(53.3)$ & 0.395 \\
\hline Weight, $\operatorname{kg}(\bar{x} \pm s)$ & $4.7 \pm 0.8$ & $4.7 \pm 0.8$ & 0.475 \\
\hline \multicolumn{4}{|l|}{$\begin{array}{l}\text { Laboratory } \\
\text { examination }(\bar{x} \pm s)\end{array}$} \\
\hline ALT (U/L) & $107.0 \pm 71.9$ & $99.2 \pm 80.6$ & 0.310 \\
\hline AST (U/L) & $158.8 \pm 120.0$ & $176.4 \pm 125.6$ & 0.151 \\
\hline ALB (g/L) & $39.3 \pm 4.5$ & $38.1 \pm 5.7$ & 0.021 \\
\hline TBIL ( $\mu \mathrm{mol} / \mathrm{L})$ & $160.0 \pm 91.5$ & $160.9 \pm 41.7$ & 0.899 \\
\hline DBIL ( $\mu \mathrm{mol} / \mathrm{L})$ & $102.1 \pm 31.4$ & $114.5 \pm 121.6$ & 0.196 \\
\hline GGT (U/L) & $668.0 \pm 550.8$ & $692.5 \pm 557.6$ & 0.658 \\
\hline APRI & $1.463 \pm 1.208$ & $1.910 \pm 1.805$ & 0.002 \\
\hline $\mathrm{Hb}(\mathrm{g} / \mathrm{L})$ & $100.3 \pm 18.1$ & $98.0 \pm 15.0$ & 0.162 \\
\hline INR & $0.974 \pm 0.195$ & $1.024 \pm 0.214$ & 0.016 \\
\hline TBA ( $\mu \mathrm{mol} / \mathrm{L})$ & $143.9 \pm 107.8$ & $146.3 \pm 71.4$ & 0.783 \\
\hline $\begin{array}{l}\text { Extrahepatic } \\
\text { malformation, } n(\%)\end{array}$ & $19(11.4)$ & $28(11.0)$ & 0.899 \\
\hline CMV infection, $n(\%)$ & 54 (32.3) & 85 (33.3) & 0.831 \\
\hline Glucocorticoid, $n$ (\%) & $122(73.1)$ & 149 (58.4) & 0.002 \\
\hline 1-month JC, $n(\%)$ & $13(7.8)$ & $17(6.7)$ & 0.662 \\
\hline 3-months JC, $n$ (\%) & 70 (41.9) & $123(48.2)$ & 0.203 \\
\hline 6-months JC, $n$ (\%) & $92(55.1)$ & $159(62.4)$ & 0.137 \\
\hline 1-year JC, $n(\%)$ & $92(55.1)$ & $158(62.0)$ & 0.160 \\
\hline 2-years JC, $n$ (\%) & $84(50.3)$ & $137(53.7)$ & 0.491 \\
\hline 1-year NLS, $n$ (\%) & $131(78.4)$ & 198 (77.6) & 0.847 \\
\hline 2-years NLS, $n(\%)$ & $96(57.5)$ & $143(56.1)$ & 0.776 \\
\hline 2-years LT, n (\%) & 32 (19.2) & 47 (18.4) & 0.851 \\
\hline 2-years death, $n(\%)$ & 39 (23.4) & $64(25.1)$ & 0.683 \\
\hline
\end{tabular}

$A L B$, albumin; $A L T$, alanine aminotransferase; APRI, aspartate aminotransferase-toplatelet ratio index; AST, aspartate aminotransferase; CMV, cytomegalovirus; DBIL, direct bilirubin; GGT, $\gamma$-glutamyl transpeptidase; Hb, hemoglobin; INR, international normalized ratio; JC, jaundice clearance; $L T$, liver transplantation; NLS, native liver survival; TBA, total bile acid; TBIL, total bilirubin. $P$ values less than 0.05 were presented in bold. 


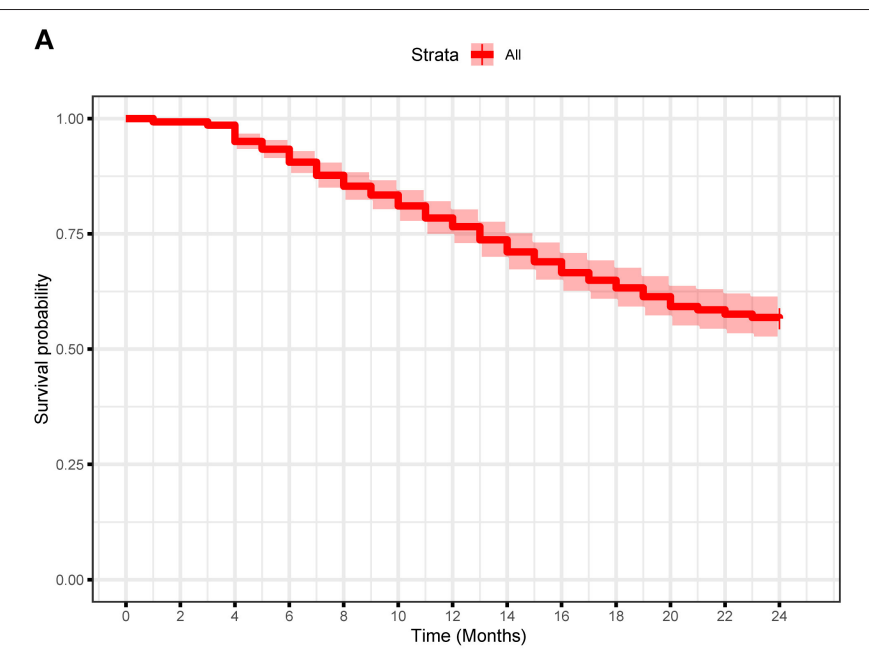

Number at risk

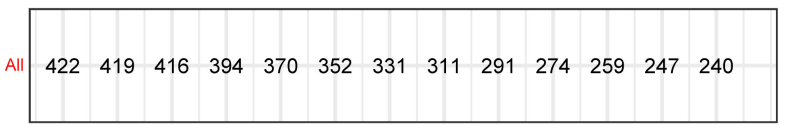

B

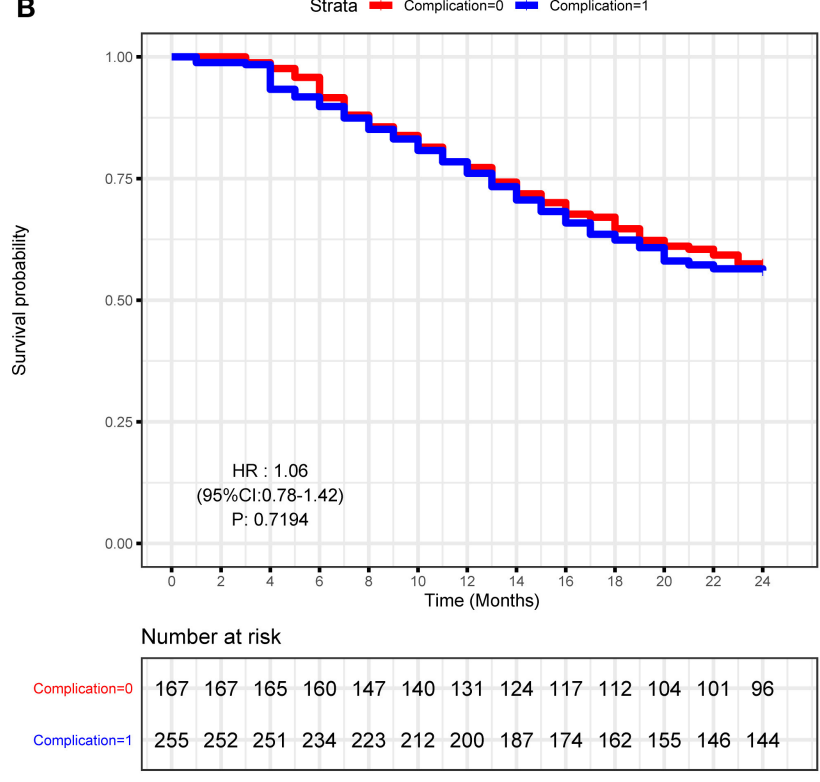

FIGURE 2 | Kaplan-Meier curve of the study cohort of biliary atresia (BA) patients. (A) patients with or without perioperative complications.

\section{Characteristics of Perioperative Complications in the BA Study Cohort}

In total, 255 patients $(60.4 \%)$ had perioperative complications (Figure 1A). Two hundred forty-three patients (57.6\%) suffered from one to three different types of complications per patient (Figure 1B), and only 12 patients (2.8\%) suffered from four to seven different types of complications per patient. Furthermore, perioperative complications were divided into DCs and SCs; 246 patients (58.3\%) had DCs, which mainly consisted of hypoalbuminemia, abnormal coagulation, ascites, and cholangitis (Figure 1C). However, only 29 patients $(6.9 \%)$ suffered from SCs, which included intestinal obstruction, anastomotic fistula, hemorrhage, and chyle leakage (Figure 1D).

\section{Comparison Between BA Patients With and Without Perioperative Complications}

To understand the difference between BA patients with and without perioperative complications, we compared their characteristics from the basic information, malformation, laboratory examination, treatment, and prognosis (Table 2). The results showed that patients with perioperative complications had lower serum ALB level, but higher APRI and INR levels when compared with those without perioperative complications (ALB, $P<0.05$; APRI, $P<0.01$; INR, $P<0.05$ ). Moreover, perioperative complications were correlated with glucocorticoid administration $(P=0.002)$; less glucocorticoids were administered in the patients with perioperative complications (odds ratio $=0.518,95 \% \mathrm{CI}=0.340-0.791$ ). We focused on the correlation between perioperative complication and prognosis
(NLS), and no significant differences were observed between the two groups $(P>0.05)$.

\section{Predictors for 2-Years Native Liver Survival of BA}

The survival curve of 2-years NLS is shown in Figure 2A. In this study, NLS was compared based on the presence of perioperative complication; Kaplan-Meier survival analysis also showed that BA patients with complications, and those without had comparable 2-years NLS ( $P=0.551$, Figure 2B). Therefore, perioperative complication was not a predictor for the 2 -years NLS in BA.

To further explore the crucial factors that affected the 2-years NLS in BA, we first performed the univariate Cox regression analysis (Table 3). To avoid missing potential risk factors, we set the $P$ threshold at 0.2 . The results revealed that age, weight, APRI, glucocorticoid, and JC (1, 3, 6, and 12 months) were potential risk factors for 2-years NLS. Subsequently, four prediction models were established by the combination of age, weight, APRI, glucocorticoid, and JC at different time points after KPE (Table 4). The results showed JC was an independent protective factor for 2-years NLS (1-month JC, $P=0.008$, HR $=0.070$; 3-months JC, $P=0.000, \mathrm{HR}=0.060$; 6-months JC, $P=0.000$, $\mathrm{HR}=0.074 ; 12$-months JC, $P=0.000, \mathrm{HR}=0.051)$. To validate the appropriate model in predicting 2-years NLS, we compared concordance indexes ( $\mathrm{C}$ indexes) and found they were 0.62 , $0.76,0.81$, and 0.83 in the prediction models with 1 -month JC, 3-months JC, 6-months JC, and 12-months JC, respectively (Table 4). Given the timeliness of the prediction model, we believed that the model with 6-months JC was the most effective in predicting 2-years NLS (C index: 0.81). Kaplan-Meier survival 
TABLE 3 | Univariate Cox regression analysis of predictors for 2-years native liver survival in biliary atresia $(n=422)$.

\begin{tabular}{|c|c|c|c|}
\hline \multirow[t]{2}{*}{ Variable } & \multicolumn{3}{|c|}{ 2-years NLS } \\
\hline & $P$-value & HR & $95 \% \mathrm{Cl}$ \\
\hline Age & 0.161 & 1.006 & $0.998-1.014$ \\
\hline Gender & 0.541 & 1.095 & $0.819-1.462$ \\
\hline Weight & 0.012 & 1.251 & $1.050-1.490$ \\
\hline ALT & 0.556 & 0.999 & $0.998-1.001$ \\
\hline AST & 0.378 & 1.000 & $0.999-1.002$ \\
\hline ALB & 0.870 & 1.002 & $0.974-1.032$ \\
\hline TBIL & 0.510 & 0.999 & 0.997-1.002 \\
\hline DBIL & 0.498 & 0.999 & $0.996-1.002$ \\
\hline GGT & 0.751 & 1.000 & $1.000-1.000$ \\
\hline $\mathrm{Hb}$ & 0.848 & 1.001 & $0.992-1.009$ \\
\hline INR & 0.888 & 1.046 & $0.560-1.952$ \\
\hline TBA & 0.791 & 1.000 & $0.999-1.002$ \\
\hline APRI & 0.029 & 1.095 & $1.009-1.189$ \\
\hline Extrahepatic malformation & 0.439 & 0.825 & $0.507-1.342$ \\
\hline CMV infection & 0.824 & 0.965 & $0.708-1.316$ \\
\hline Complication & 0.725 & 1.055 & $0.784-1.420$ \\
\hline Glucocorticoid & 0.000 & 1.992 & $1.419-2.797$ \\
\hline 1-month JC & 0.004 & 0.055 & $0.008-0.395$ \\
\hline 3-months JC & 0.000 & 0.059 & $0.034-0.102$ \\
\hline 6-months JC & 0.000 & 0.079 & $0.054-0.114$ \\
\hline 1-year JC & 0.000 & 0.054 & $0.036-0.081$ \\
\hline
\end{tabular}

$A L B$, albumin; $A L T$, alanine aminotransferase; $A P R I$, aspartate aminotransferase-toplatelet ratio index; AST, aspartate aminotransferase; Cl, confidence interval; CMV, cytomegalovirus; DBIL, direct bilirubin; GGT, $\gamma$-glutamyl transpeptidase; Hb, hemoglobin; $H R$, hazard ratio; INR, international normalized ratio; JC, jaundice clearance; $L T$, liver transplantation; NLS, native liver survival; TBA, total bile acid; TBIL, total bilirubin.

analysis also supported the above findings that BA patients with JC had a remarkably higher 2-years NLS than those with jaundice $(P<0.01)$ (Figure 3).

\section{DISCUSSION}

To our knowledge, this is the first large study that analyzed perioperative complications after KPE for patients with type III BA. In our study, the incidence and mortality rates of perioperative complications were 60.4 and $0.7 \%$, respectively, which were much lower compared to the findings that were presented previously (96.8 and $2 \%$, respectively) $(14,21)$. This may be related to the fact that our institution, the largest BA center in China, is highly experienced in performing KPE. In a multicenter study in Europe, it was also observed that high caseload benefited outcomes $(22,23)$. Most patients $(243,57.6 \%)$ had one to three different types of complications, and DCs were the most prevalent $(246,58.3 \%)$. The most common type of DCs was hypoalbuminemia, followed by abnormal coagulation, ascites, cholangitis, portal hypertension, and hepatic cysts. The incidence of perioperative cholangitis was low (8.5\%), which was potentially due to the short perioperative observation period of 30 days. Notably, recurrent cholangitis can lead to a lower
TABLE 4 | Multivariate Cox regression analysis of predictors for 2-years native liver survival in biliary atresia $(n=422)$.

\begin{tabular}{|c|c|c|c|c|}
\hline \multirow[t]{2}{*}{ Variable } & \multicolumn{3}{|c|}{ 2-years NLS } & \multirow[t]{2}{*}{ C index } \\
\hline & $\begin{array}{l}\text { Adjusted } \\
P \text {-value }\end{array}$ & $\begin{array}{c}\text { Adjusted } \\
\text { HR }\end{array}$ & Adjusted $95 \% \mathrm{Cl}$ & \\
\hline \multicolumn{5}{|l|}{ Model 1} \\
\hline Age & 0.629 & 1.003 & $0.992-1.014$ & 0.62 \\
\hline Weight & 0.050 & 1.267 & $1.000-1.606$ & \\
\hline APRI & 0.206 & 1.057 & $0.970-1.151$ & \\
\hline Glucocorticoid & 0.003 & 1.697 & $1.192-2.416$ & \\
\hline 1-month JC & 0.008 & 0.070 & $0.010-0.504$ & \\
\hline \multicolumn{5}{|l|}{ Model 2} \\
\hline Age & 0.452 & 0.996 & $0.985-1.007$ & 0.76 \\
\hline Weight & 0.098 & 1.230 & $0.962-1.572$ & \\
\hline APRI & 0.098 & 1.072 & $0.987-1.163$ & \\
\hline Glucocorticoid & 0.869 & 1.031 & $0.720-1.476$ & \\
\hline 3-months JC & 0.000 & 0.060 & $0.034-0.105$ & \\
\hline \multicolumn{5}{|l|}{ Model 3} \\
\hline Age & 0.535 & 0.997 & $0.986-1.007$ & 0.81 \\
\hline Weight & 0.016 & 1.351 & $1.057-1.726$ & \\
\hline APRI & 0.165 & 1.065 & $0.974-1.164$ & \\
\hline Glucocorticoid & 0.428 & 0.862 & $0.596-1.245$ & \\
\hline 6-months JC & 0.000 & 0.074 & $0.050-0.110$ & \\
\hline \multicolumn{5}{|l|}{ Model 4} \\
\hline Age & 0.936 & 1.000 & $0.989-1.011$ & 0.83 \\
\hline Weight & 0.117 & 1.218 & $0.951-1.560$ & \\
\hline APRI & 0.042 & 1.098 & $1.004-1.202$ & \\
\hline Glucocorticoid & 0.363 & 0.843 & $0.584-1.218$ & \\
\hline 1-year JC & 0.000 & 0.051 & $0.033-0.078$ & \\
\hline
\end{tabular}

APRI, aspartate aminotransferase-to-platelet ratio index; JC, jaundice clearance; NLS, native liver survival.

NLS (24). Patients who experience a fever and worsening jaundice need to be vigilant of cholangitis $(24,25)$, and the use of antireflux valves, jejunal Roux-en-Y loop and antibiotics may help to reduce the occurrence of cholangitis $(25,26)$. In a recent study in 2015, it was shown that SCs affected $41 \%$ of 153 patients (27), which was much higher than our results $(6.9 \%)$. In our study cohort, we did observe hemorrhage, anastomotic fistula, intestinal obstruction, and chyle leakage; however, hernia and abscess complications that have been reported in previous studies were not observed (27). Our data showed that intestinal complications (intestinal obstruction and anastomotic fistula) were the most frequent SCs, which was consistent with the findings presented in other reports (14). After KPE, patients with acute abdominal pain must immediately and carefully be assessed for intestinal complications. In order to reduce intestinal complications, care must be taken to accurately close the mesenteric defect and to not twist the intestinal loop during KPE (28). The incidence of hemorrhage was $3.1 \%$ without massive bleeding or hemorrhagic shock, which was lower than the findings reported previously (5\%) (27). Correction of coagulation and protection of blood vessels can 

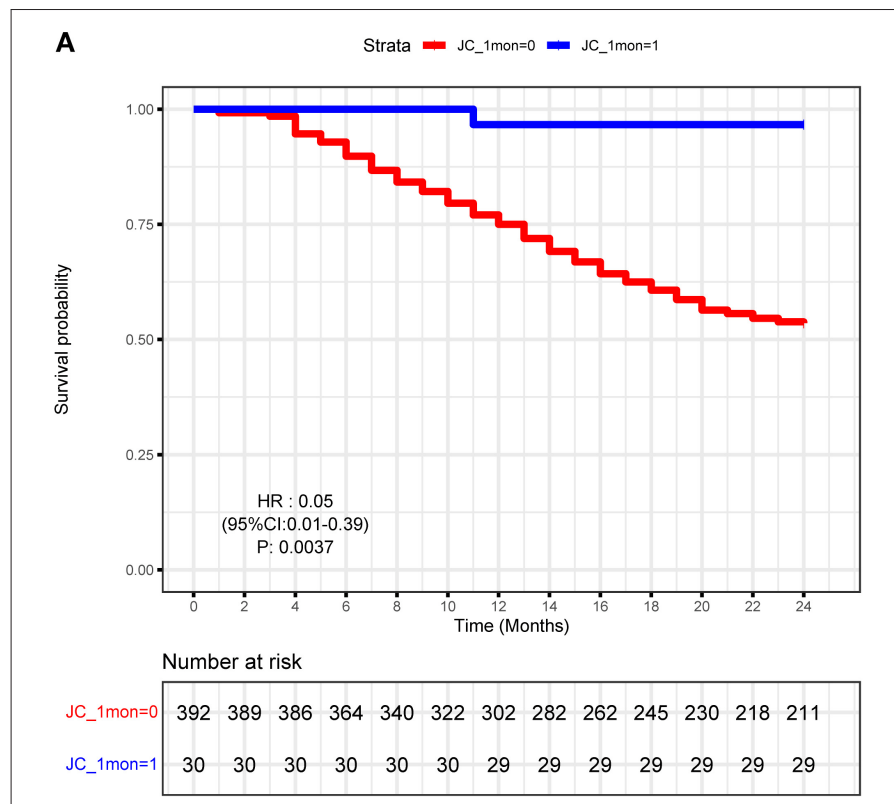

C
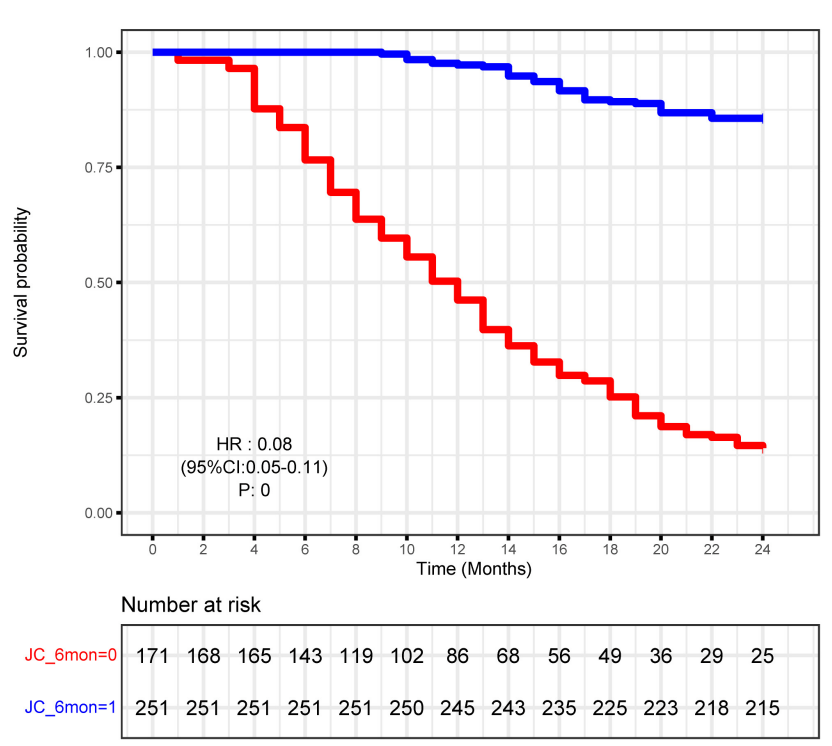

FIGURE 3 | Kaplan-Meier curve of biliary atresia (BA) patients with or without JC.

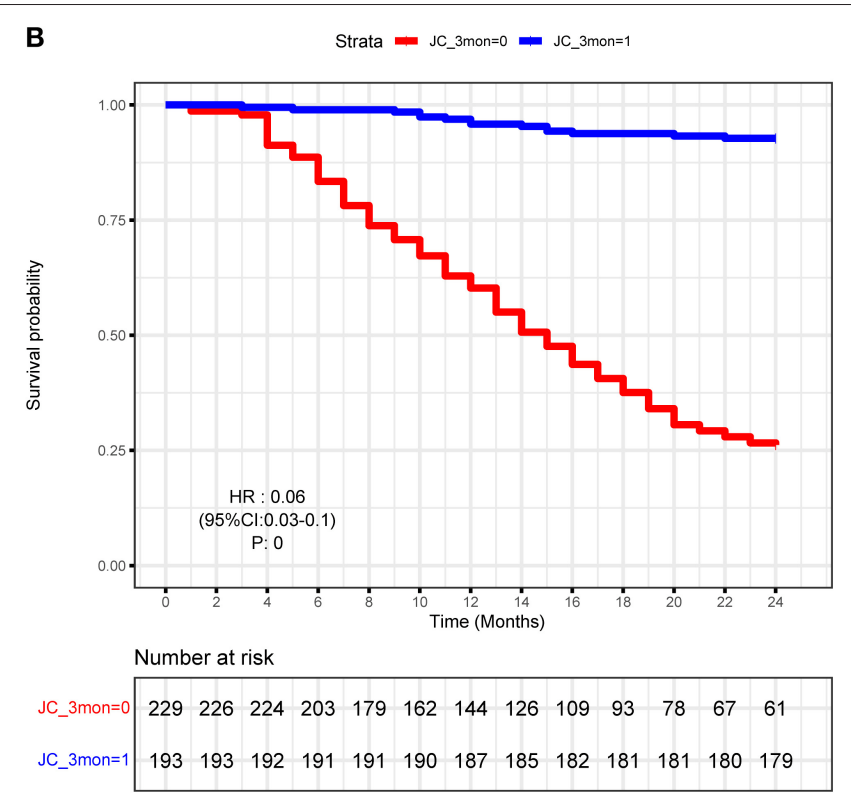

D

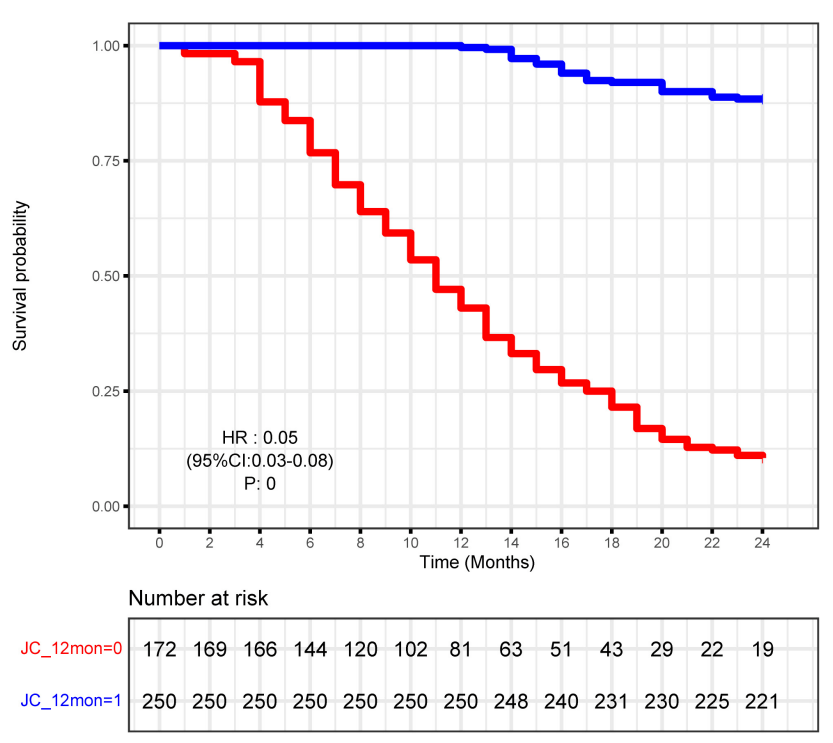

reduce hemorrhage. In this study, serum ALB concentration, INR, and APRI had a significant change in BA patients with perioperative complications when compared with those without complications, which indicated that liver synthetic functions or fibrosis may be risk factors for disease-related perioperative complications in BA.

In previous studies, it has been shown that the short-term (1-3 years) NLS rate of BA ranged from 20.3 to $75.8 \%$ (10). Consistent with this, in our study, the 1-year and 2-years NLS rates were 78.0 and $56.6 \%$, respectively. Although perioperative complications were common in BA patients, they were not predictors for 2-years NLS, which suggested perioperative complications had no impact on the BA prognosis. However, JC was considered a crucial predictor for improved NLS (11, 29-32). In a large study in Japan, it was demonstrated that TBIL $<17.1 \mu \mathrm{mol} / \mathrm{L}$ at 3 months post-KPE predicted an improved NLS (33). Moreover, in another multicenter study in the United States, it was shown that TBIL $<34.2 \mu \mathrm{mol} / \mathrm{L}$ at 3 months after $\mathrm{KPE}$ was a predictor for improved NLS (34). In this study, the JC (TBIL $<20 \mu \mathrm{mol} / \mathrm{L}$ ) rate was also highly predictive for 2-years NLS. Furthermore, we evaluated the accuracy of prediction models by comparing $\mathrm{C}$ index and found the prediction model with 6-months JC 
was more appropriate than other models with 1-month JC, 3months JC, and 12-months JC, which may be explained by the highest level at 6 months after KPE. Taken together, these findings suggested that JC after KPE resulted in a good bile flow with better NLS.

Treatment with adjuvant steroids after KPE has previously been used to assist restoring bile flow and anti-inflammation; however, the effects of steroids on BA outcome remain controversial $(35,36)$. In several studies, no benefits of postoperative steroids on prognosis were observed $(36,37)$, whereas other studies showed that treatment with adjuvant steroids after KPE may improve short-term ( $\leq 1$ year) JC. In this study, our results revealed that patients treated with adjuvant steroids initially suffered from no postoperative bile drainage and also had a higher risk obtaining a low 2 -years NLS (HR $=1.992$, 95\% CI $=1.419-2.797, P=0.000)$. The paradoxical effects of the use of steroids on the prognosis of BA remain to be elucidated.

In this study, the average age at KPE was $62.9 \pm 18.4$ days, which was similar to that published in international journals (38). A young age, especially $<60$ days old at KPE, has been widely, although not universally, linked to improved outcome in BA, which may be explained by the milder liver fibrosis in younger patients (16, 39-41). However, Song et al. (42) demonstrated that BA patients younger than 40 days had a poorer NLS rate and that, in these patients, severe liver fibrosis may account for the reverse consequence $(35,39)$. Interestingly, our results showed that age at KPE had no significant impact on prognosis of BA. Despite the controversy, it has been advised that infants with cholestasis need to be evaluated and appropriately managed on time and that when BA is diagnosed KPE should be performed as soon as possible (43-45).

Aspartate aminotransferase-to-platelet ratio index reflects portal fibrogenesis and could diagnose liver fibrosis in BA (40, 46). Ihn et al. (29) demonstrated that APRIs at month 4, GGT at month 5, and JC at month 6 after KPE were independent risk factors for NLS. Grieve et al. (47) also supported the role of APRI at KPE in predicting NLS using univariate analysis. Our results showed that APRI at KPE had significance in predicting NLS without considering other factors, but exerted a modest effect in the prediction model for NLS. Therefore, monitoring the change of APRI may be valuable in predicting NLS in BA.

Studies in the United Kingdom and Germany $(48,49)$ demonstrated that approximately $10 \%$ of BA patients were infected with CMV. However, a much higher incidence of CMV infection (34-69\%) was reported in Brazil, Sweden, Pakistan, China, and South Africa (50-54). In the current study, CMV infection was present in 139 patients (32.9\%). The varied incidence of CMV infection may be explained by the different racial background (55). Hepatotropic viral infections have been proposed as a causative mechanism of action for bile duct and immune destruction (56). Liver T-cell responses to CMV and deficiency of regulatory $\mathrm{T}$ cells in BA aggravated inflammation and autoreactivity, which potentially allowed for exaggerated bile duct injury (57). Zani et al. (49) found that BA patients with positive CMV IgM had lower JC and NLS. However, our results indicated that CMV infection had little impact on prognosis, which was consistent with the findings presented in two German studies $(48,58)$. The above results suggested a controversial role of CMV infection in BA. In line with the data of Zani et al. (49), our results also indicated that CMV-positive BA patients were significantly older than those who were CMV negative (CMV negative: $60.69 \pm 18.43$ days; CMV positive: $67.36 \pm 17.70$ days; $P<0.001$ ), implying that CMV infection was simply a secondary phenomenon rather than a factor involved in the etiology of BA. Therefore, our data demonstrated that CMV may not play a major role in the pathogenesis of BA, and further studies are needed in the future.

This was a single-center retrospective study, which was considered a limitation. Nevertheless, strict inclusion and exclusion criteria were adopted, and all surgeries were performed by the same surgical team, which would increase the consistency and could be regarded as an advantage. A 2-years NLS rate represented a short-term prognosis when compared with the 10-20-years results that have been reported in other studies. However, in this study, multivariate analysis models were applied, and important predictors for prognosis were identified, including age, weight, APRI, glucocorticoids, and JC. Notwithstanding these limitations, this study, in which a large sample size was used, comprehensively revealed the characteristics of BA perioperative complications and prognosis, thereby providing the scope for future research in perioperative management.

In conclusion, perioperative complication was common in BA, but was not a predictor for 2-years NLS. However, 6-months JC after KPE was an independent protective factor for 2-years NLS. Therefore, monitoring the dynamic change of serum TBIL and taking the corresponding treatments after KPE may be crucial in improving the survival rate of native liver in BA.

\section{DATA AVAILABILITY STATEMENT}

All datasets generated for this study are included in the article/supplementary material.

\section{ETHICS STATEMENT}

The studies involving human participants were reviewed and approved by Children's Hospital of Fudan University. Written informed consent to participate in this study was provided by the participants' legal guardian/next of kin. Written informed consent was obtained from the minor(s)' legal guardian/next of kin for the publication of any potentially identifiable images or data included in this article.

\section{AUTHOR CONTRIBUTIONS}

SZ and RD conceived and designed the study. MD and JW collected the clinical data, performed data analysis, and wrote the paper. YT and JJ offered the assist in data collection. GC, YH, and $\mathrm{ZS}$ reviewed and edited the manuscript. All authors read and approved the manuscript. 


\section{FUNDING}

This study received financial support from Shanghai Key Disciplines (no. 2017ZZ02022), Shanghai Municipal Key Clinical Specialty (no. shslczdzk05703), National Natural Science

\section{REFERENCES}

1. Asai A, Miethke A, Bezerra JA. Pathogenesis of biliary atresia: defining biology to understand clinical phenotypes. Nat Rev Gastroenterol Hepatol. (2015) 12:342-52. doi: 10.1038/nrgastro.2015.74

2. Bezerra JA, Wells RG, Mack CL, Karpen SJ, Hoofnagle JH, Doo E, et al. Biliary atresia: clinical and research challenges for the 21(st) Century. Hepatology. (2018) 68:1163-73. doi: 10.1002/hep.29905

3. Nio M. Japanese biliary atresia registry. Pediatr Surg Int. (2017) 33:1319-25. doi: 10.1007/s00383-017-4160-x

4. Lakshminarayanan B, Davenport M. Biliary atresia: a comprehensive review. J Autoimmun. (2016) 73:1-9. doi: 10.1016/j.jaut.2016.06.005

5. Nio M, Wada M, Sasaki H, Tanaka H, Okamura A. Risk factors affecting latepresenting liver failure in adult patients with biliary atresia. J Pediatr Surg. (2012) 47:2179-83. doi: 10.1016/j.jpedsurg.2012.09.003

6. Superina R, Magee JC, Brandt ML, Healey PJ, Tiao G, Ryckman F, et al. The anatomic pattern of biliary atresia identified at time of Kasai hepatoportoenterostomy and early postoperative clearance of jaundice are significant predictors of transplant-free survival. Ann Surg. (2011) 254:57785. doi: 10.1097/SLA.0b013e3182300950

7. Nightingale S, Stormon MO, O'Loughlin EV, Shun A, Thomas G, Benchimol EI, et al. Early posthepatoportoenterostomy predictors of native liver survival in biliary atresia. J Pediatr Gastroenterol Nutr. (2017) 64:203-9. doi: 10.1097/MPG.0000000000001289

8. Superina R. Biliary atresia and liver transplantation: results and thoughts for primary liver transplantation in select patients. Pediatr Surg Int. (2017) 33:1297-304. doi: 10.1007/s00383-017-4174-4

9. Zhou Y, Yin Z, Hou B, Yu M, Chen R, Jin H, et al. Expression profiles and prognostic significance of RNA N6-methyladenosine-related genes in patients with hepatocellular carcinoma: evidence from independent datasets. Cancer Manag Res. (2019) 11:3921-31. doi: 10.2147/CMAR. S191565

10. Jimenez-Rivera C, Jolin-Dahel KS, Fortinsky KJ, Gozdyra P, Benchimol EI. International incidence and outcomes of biliary atresia. J Pediatr Gastroenterol Nutr. (2013) 56:344-54. doi: 10.1097/MPG.0b013e318282a913

11. Sundaram SS, Mack CL, Feldman AG, Sokol RJ. Biliary atresia: indications and timing of liver transplantation and optimization of pretransplant care. Liver Transpl. (2017) 23:96-109. doi: 10.1002/lt.24640

12. Eboli L, Tannuri AC, Gibelli N, Silva T, Braga P, Tannuri U. Comparison of the results of living donor liver transplantation due to acute liver failure and biliary atresia in a quaternary center. Transplant Proc. (2017) 49:832-5. doi: 10.1016/j.transproceed.2017.03.001

13. Wong KKY, Wong CWY. A review of long-term outcome and quality of life of patients after Kasai operation surviving with native livers. Pediatr Surg Int. (2017) 33:1283-7. doi: 10.1007/s00383-017-4158-4

14. Wilde JCH, Calinescu AM, Wildhaber BE. Perioperative complications in neonatal surgery: biliary atresia and choledochal malformations. Eur J Pediatr Surg. (2018) 28:156-62. doi: 10.1055/s-0038-1636929

15. Sanchez-Valle A, Kassira N, Varela VC, Radu SC, Paidas C, Kirby RS. Biliary atresia: epidemiology, genetics, clinical update, and public health perspective. Adv Pediatr. (2017) 64:285-305. doi: 10.1016/j.yapd.2017.03.012

16. Pakarinen MP, Johansen LS, Svensson JF, Bjornland K, Gatzinsky V, Stenstrom $\mathrm{P}$, et al. Outcomes of biliary atresia in the Nordic countries - a multicenter study of 158 patients during 2005-2016. J Pediatr Surg. (2018) 53:1509-15. doi: 10.1016/j.jpedsurg.2017.08.048

17. Wong CWY, Chung PHY, Tam PKH, Wong KKY. Long-term results and quality of life assessment in biliary atresia patients: a 35-year experience in a tertiary hospital. J Pediatr Gastroenterol Nutr. (2018) 66:570-4. doi: 10.1097/MPG.0000000000001854
Foundation of China (nos 81770519, 81771633, 81873545, and 81974059), The Science Foundation of Shanghai (nos. 18411969100 and 19ZR1406600), and Children's National Medical Center (nos. EK1125180104, EKYY20180204, EK112520180211, and EK112520180310).

18. Grisotti G, Cowles RA. Complications in pediatric hepatobiliary surgery. Semin Pediatr Surg. (2016) 25:388-94. doi: 10.1053/j.sempedsurg.2016.10.004

19. Zhu F, Li Y, Guo Z, Cao L, Feng D, Zhang T, et al. Nomogram to predict postoperative intra-abdominal septic complications after bowel resection and primary anastomosis for crohn's disease. Dis Colon Rectum. (2020) 63:629-38. doi: 10.1097/DCR.0000000000001602

20. Koga H, Wada M, Nakamura H, Miyano G, Okawada M, Lane GJ, et al. Factors influencing jaundice-free survival with the native liver in postportoenterostomy biliary atresia patients: results from a single institution. $J$ Pediatr Surg. (2013) 48:2368-72. doi: 10.1016/j.jpedsurg.2013.08.007

21. Odievre M, Valayer J, Razemon-Pinta M, Habib EC, Alagille D. Hepatic porto-enterostomy or cholecystostomy in the treatment of extrahepatic biliary atresia. a study of 49 cases. J Pediatr. (1976) 88:774-9. doi: 10.1016/S0022-3476(76)81113-4

22. Wong $\mathrm{ZH}$, Davenport M. What happens after kasai for biliary atresia? a European multicenter survey. Eur J Pediatr Surg. (2019) 29:1-6. doi: 10.1055/s-0038-1668146

23. Ihn $\mathrm{K}, \mathrm{Na} \mathrm{Y}$, Ho IG, Lee D, Koh H, Han SJ. A periodic comparison of the survival and prognostic factors of biliary atresia after Kasai portoenterostomy: a single-center study in Korea. Pediatr Surg Int. (2019) 35:285-92. doi: 10.1007/s00383-018-04434-5

24. Chung PH, Wong KK, Tam PK. Predictors for failure after Kasai operation. J Pediatr Surg. (2015) 50:293-6. doi: 10.1016/j.jpedsurg.2014.11.015

25. Luo Y, Zheng S. Current concept about postoperative cholangitis in biliary atresia. World J Pediatr. (2008) 4:14-9. doi: 10.1007/s12519-008-0003-0

26. Nio M, Wada M, Sasaki H, Kazama T, Tanaka H, Kudo H. Technical standardization of Kasai portoenterostomy for biliary atresia. J Pediatr Surg. (2016) 51:2105-8. doi: 10.1016/j.jpedsurg.2016.09.047

27. Madadi-Sanjani O, Carl N, Longerich T, Petersen C, Andruszkow JH. Inguinal hernias represent the most frequent surgical complication after kasai in biliary atresia infants. Biomed Res Int. (2015) 2015:383791. doi: 10.1155/2015/383791

28. Eberhardt CS, Merlini L, McLin VA, Wildhaber BE. Cholestasis as the leading sign of a transmesenteric hernia in a split-liver transplanted child a case report and review of literature. Pediatr Transplant. (2012) 16:E172-6. doi: 10.1111/j.1399-3046.2011.01496.x

29. Ihn K, Ho IG, Chang EY, Han SJ. Correlation between gamma-glutamyl transpeptidase activity and outcomes after Kasai portoenterostomy for biliary atresia. J Pediatr Surg. (2018) 53:461-7. doi: 10.1016/j.jpedsurg.2017.10.001

30. Wang Z, Chen Y, Peng C, Pang W, Zhang T, Wu D, et al. Five-year native liver survival analysis in biliary atresia from a single large Chinese center: the death/liver transplantation hazard change and the importance of rapid early clearance of jaundice. J Pediatr Surg. (2018) 54:1680-5. doi: 10.1016/j.jpedsurg.2018.09.025

31. Nakajima H, Koga H, Okawada M, Nakamura H, Lane GJ, Yamataka A. Does time taken to achieve jaundice-clearance influence survival of the native liver in post-Kasai biliary atresia? World J Pediatr. (2018) 14:191-6. doi: 10.1007/s12519-018-0139-5

32. Redkar R, Karkera PJ, Raj V, Bangar A, Hathiramani V, Krishnan J. Outcome of biliary atresia after kasai's portoenterostomy: a 15-year experience. Indian Pediatr. (2017) 54:291-4. doi: 10.1007/s13312-017-1091-5

33. Ohhama Y, Shinkai M, Fujita S, Nishi T, Yamamoto H. Early prediction of long-term survival and the timing of liver transplantation after the Kasai operation. J Pediatr Surg. (2000) 35:1031-4. doi: 10.1053/jpsu.2000.7765

34. Shneider BL, Brown MB, Haber B, Whitington PF, Schwarz K, Squires R, et al. A multicenter study of the outcome of biliary atresia in the United States, 1997 to 2000. J Pediatr. (2006) 148:467-74. doi: 10.1016/j.jpeds.2005.12.054

35. Webb NL, Jiwane A, Ooi CY, Nightinghale S, Adams SE, Krishnan U. Clinical significance of liver histology on outcomes in biliary atresia. J Paediatr Child Health. (2017) 53:252-6. doi: 10.1111/jpc.13371 
36. Tyraskis A, Parsons C, Davenport M. Glucocorticosteroids for infants with biliary atresia following Kasai portoenterostomy. Cochrane Database Syst Rev. (2018) 5:CD008735. doi: 10.1002/14651858.CD008735.pub3

37. Sarkhy A, Schreiber RA, Milner RA, Barker CC. Does adjuvant steroid therapy post-Kasai portoenterostomy improve outcome of biliary atresia? systematic review and meta-analysis. Can J Gastroenterol. (2011) 25:440-4. doi: 10.1155/2011/125610

38. Garcia AV, Ladd MR, Crawford T, Culbreath K, Tetteh O, Alaish SM, et al. Analysis of risk factors for morbidity in children undergoing the Kasai procedure for biliary atresia. Pediatr Surg Int. (2018) 34:837-44. doi: 10.1007/s00383-018-4298-1

39. Moyer K, Kaimal V, Pacheco C, Mourya R, Xu H, Shivakumar P, et al. Staging of biliary atresia at diagnosis by molecular profiling of the liver. Genome Med. (2010) 2:33. doi: 10.1186/gm154

40. Suominen JS, Lampela H, Heikkila P, Lohi J, Jalanko H, Pakarinen MP. APRi predicts native liver survival by reflecting portal fibrogenesis and hepatic neovascularization at the time of portoenterostomy in biliary atresia. J Pediatr Surg. (2015) 50:1528-31. doi: 10.1016/j.jpedsurg.2014.11.046

41. Weerasooriya VS, White FV, Shepherd RW. Hepatic fibrosis and survival in biliary atresia. J Pediatr. (2004) 144:123-5. doi: 10.1016/j.jpeds.2003.09.042

42. Song Z, Dong R, Shen Z, Chen G, Yang Y, Zheng S. Surgical outcome and etiologic heterogeneity of infants with biliary atresia who received Kasai operation less than 60 days after birth: a retrospective study. Medicine (Baltimore). (2017) 96:e7267. doi: 10.1097/MD.0000000000007267

43. Lin JS, Chen SC, Lu CL, Lee HC, Yeung CY, Chan WT. Reduction of the ages at diagnosis and operation of biliary atresia in Taiwan: a 15-year population-based cohort study. World J Gastroenterol. (2015) 21:13080-6. doi: 10.3748/wjg.v21.i46.13080

44. Townsend MR, Jaber A, Abi Nader H, Eid SM, Schwarz K. Factors associated with timing and adverse outcomes in patients with biliary atresia undergoing kasai hepatoportoenterostomy. J Pediatr. (2018) 199:237-42.e32. doi: 10.1016/j.jpeds.2018.04.001

45. Hopkins PC, Yazigi N, Nylund CM. Incidence of biliary atresia and timing of hepatoportoenterostomy in the United States. J Pediatr. (2017) 187:253-7. doi: 10.1016/j.jpeds.2017.05.006

46. Yang LY, Fu J, Peng XF, Pang SY, Gao KK, Chen ZR, et al. Validation of aspartate aminotransferase to platelet ratio for diagnosis of liver fibrosis and prediction of postoperative prognosis in infants with biliary atresia. World $J$ Gastroenterol. (2015) 21:5893-900. doi: 10.3748/wjg.v21.i19.5893

47. Grieve A, Makin E, Davenport M. Aspartate aminotransferaseto-Platelet ratio index (APRi) in infants with biliary atresia: prognostic value at presentation. J Pediatr Surg. (2013) 48:789-95. doi: 10.1016/j.jpedsurg.2012.10.010

48. Rauschenfels S, Krassmann M, Al-Masri AN, Verhagen W, Leonhardt J, Kuebler JF, et al. Incidence of hepatotropic viruses in biliary atresia. Eur J Pediatr. (2009) 168:469-76. doi: 10.1007/s00431-008-0774-2
49. Zani A, Quaglia A, Hadzić N, Zuckerman M, Davenport M. Cytomegalovirusassociated biliary atresia: an aetiological and prognostic subgroup. $J$ Pediatr Surg. (2015) 50:1739-45. doi: 10.1016/j.jpedsurg.2015. 03.001

50. Fischler B, Ehrnst A, Forsgren M, Orvell C, Nemeth A. The viral association of neonatal cholestasis in Sweden: a possible link between cytomegalovirus infection and extrahepatic biliary atresia. J Pediatr Gastroenterol Nutr. (1998) 27:57-64. doi: 10.1097/00005176-199807000-00010

51. De Tommaso AM, Andrade PD, Costa SC, Escanhoela CA, Hessel G. High frequency of human cytomegalovirus DNA in the liver of infants with extrahepatic neonatal cholestasis. BMC Infect Dis. (2005) 5:108. doi: 10.1186/1471-2334-5-108

52. Soomro GB, Abbas Z, Hassan M, Luck N, Memon Y, Khan AW. Is there any association of extra hepatic biliary atresia with cytomegalovirus or other infections? J Pak Med Assoc. (2011) 61:281-3.

53. Moore SW, Zabiegaj-Zwick C, Nel E. Problems related to CMV infection and biliary atresia. S Afr Med J. (2012) 102:890-2. doi: 10.7196/SAMJ.6163

54. Xu Y, Yu J, Zhang R, Yin Y, Ye J, Tan L, et al. The perinatal infection of cytomegalovirus is an important etiology for biliary atresia in China. Clin Pediatr (Phila). (2012) 51:109-13. doi: 10.1177/0009922811406264

55. Parolini F, Hadzic N, Davenport M. Adjuvant therapy of cytomegalovirus IgM + ve associated biliary atresia: prima facie evidence of effect. J Pediatr Surg. (2019) 54:1941-5. doi: 10.1016/j.jpedsurg.2018.12.014

56. Lopez RN, Ooi CY, Krishnan U. Early and peri-operative prognostic indicators in infants undergoing hepatic portoenterostomy for biliary atresia: a review. Curr Gastroenterol Rep. (2017) 19:16. doi: 10.1007/s11894-017-0555-z

57. Brindley SM, Lanham AM, Karrer FM, Tucker RM, Fontenot AP, Mack CL. Cytomegalovirus-specific T-cell reactivity in biliary atresia at the time of diagnosis is associated with deficits in regulatory T cells. Hepatology. (2012) 55:1130-8. doi: 10.1002/hep.24807

58. Schukfeh N, Al-Gamrah A, Petersen C, Kuebler JF. Detection of hepatotropic viruses has no impact on the prognosis after Kasai procedure. J Pediatr Surg. (2012) 47:1828-32. doi: 10.1016/j.jpedsurg.2012.04.024

Conflict of Interest: The authors declare that the research was conducted in the absence of any commercial or financial relationships that could be construed as a potential conflict of interest.

Copyright (c) 2020 Du, Wang, Tang, Jiang, Chen, Huang, Shen, Dong and Zheng. This is an open-access article distributed under the terms of the Creative Commons Attribution License (CC BY). The use, distribution or reproduction in other forums is permitted, provided the original author(s) and the copyright owner(s) are credited and that the original publication in this journal is cited, in accordance with accepted academic practice. No use, distribution or reproduction is permitted which does not comply with these terms. 